\title{
AC 2009-575: AN ALTERNATIVE APPROACH FOR TEACHING MULTIBODY DYNAMICS
}

\section{George Sutherland, Rochester Institute of Technology}

DR. GEORGE H. SUTHERLAND is a professor in the Manufacturing \& Mechanical

Engineering Technology and Packaging Science Department at the Rochester Institute of Technology in New York State. Dr. Sutherland's technical interests include the dynamics of high speed machinery and vehicle dynamics. He was previously an associate professor in ME at Ohio State University, a manager at General Electric, a VP at CAMP Inc and President of Washington Manufacturing Services. 


\section{Alternative Approach for Teaching Multibody Dynamics}

\section{Abstract}

The paper presents an alternative method, the Implicit Constraint Approach (ICA), for developing the equations of motion to describe a system of connected bodies typified by mechanisms and robotic devices. By its simplicity of description and implementation compared to conventional methods, the ICA engages students more in the physics (rather than the mathematics) of the system under study. The ICA enables the direct formation of all needed equations by using only the dynamic equilibrium conditions (in the Newton or Lagrange form). Unlike current approaches, no auxiliary algebraic constraint equations are required. Thus the descriptive equations are ordinary differential equation (ODEs) rather than differential-algebraic equations (DAEs). This leads to simpler mathematics and a broader range of potentially useful numerical integration methods. Examples are provided which illustrate the use of the ICA. The more involved examples employ a general purpose computer program that uses the ICA to provide the kinematics and forces for a planar mechanism of arbitrary complexity.

\section{Current Methods}

If asked to find the motion properties and dynamic forces in a mechanism like a planar fourbar linkage, the typical first step is to form the closure loop equation ${ }^{1,2}$ (Figure 1). For simple problems like the planar four-bar, this typically nonlinear equation is directly solved for the unknown motion properties as a function of prescribed input properties. However for more complex problems the direct solution of the loop equations is either quite difficult or impossible and the loop equations become auxiliary constraint equations ${ }^{3}$ that must be added to the equations of motion (from the application of Newton's Second Law or the equivalent approach) for the mechanical system. Chace ${ }^{4}$ first found that using the second derivative of the loop equations was most effective in numerically solving mechanical system dynamics using a digital computer. The constraint equation derivative form can be used if the system initial conditions are met exactly and small time steps are used ${ }^{3}$. However the numerical solution will usually drift from the actual solution as the numerical integration proceeds and additional methods ${ }^{5,6}$ have been developed to keep the solution on track. $\mathrm{MSC}_{-\mathrm{ADAMS}}{ }^{7}$ is a commercial program for mechanism dynamic simulation that has evolved from the original methods of Chace ${ }^{4}$.

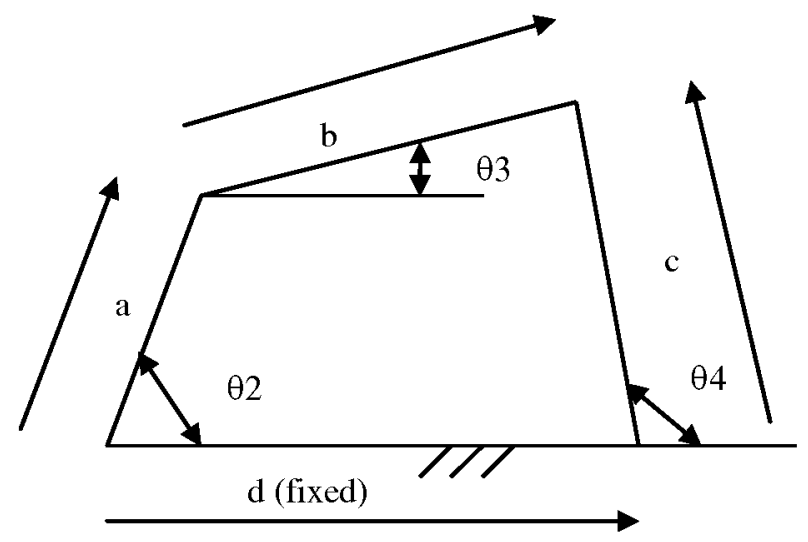

Vector loop equation

$a+b=d+c$

Figure 1 Vector Loop Equation for a Planar Four-Bar Linkage 
Implicit Constraint Approach

The underlying assumption in current mechanism analysis approaches is that the joints, which connect the moving rigid bodies, are ideal in their behavior. The concept of forming a mechanism model using a standard set of idealized joints dates back to Reuleaux ${ }^{8}$, and is still the underlying assumption in modern dynamic analysis computer programs like MSC-Adams, MathWorks SimMechanics ${ }^{9}$, and Working Model $^{10}$. When joints, like roller bearings, are rigidly mounted and preloaded, radial stiffnesses of $10^{8} \mathrm{~N} / \mathrm{m}$ and higher ${ }^{11}$ are typical and the ideal joint assumption is reasonable. However when joints are designed with clearances and flexible mounts, the ideal joint assumption might not be acceptable (depending on the analyst's need).

The Implicit Constraint Approach (ICA) ${ }^{12}$ offers an alternative approach to mechanism analysis where joint flexibility is an inherent characteristic utilized in the method. The relative kinematic characteristics of standard joints are not used as basic assumptions. Instead each joint type is characterized by the forces that are generated when the two components of the joint are displaced relative to each other. A revolute joint (in three dimensions) is characterized by three orthogonal reaction force components and two orthogonal reaction moment components. There is no reaction moment about the axis that corresponds to the direction of joint relative rotation. (In a two dimensional analysis this simplifies to just two orthogonal reaction forces acting in the plane through the revolute joint center, while the moment reaction about the axis perpendicular to the plane of motion is zero.) Using the ICA, these unknown reaction forces are assumed to be proportional to the joint component relative displacements. Although there is a more rigorous axiomatic underpinning to the $\mathrm{ICA}^{12}$, it can be visualized by considering the joint components to be connected to each other by specially-defined zero free length springs as shown for a planar four-bar linkage in Figure 2.

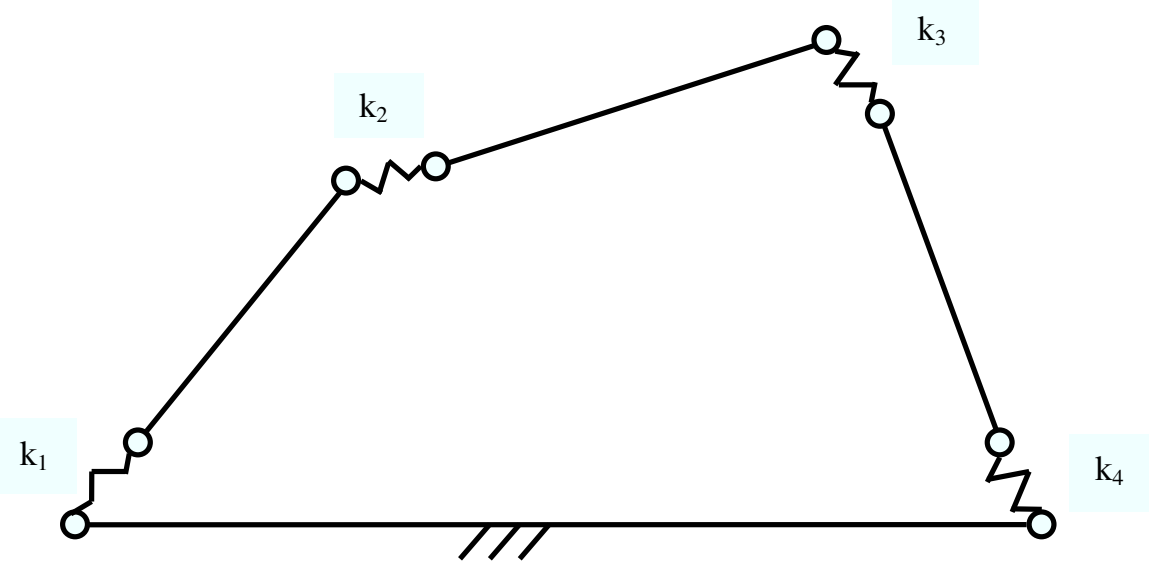

Figure 2 Revolute Joints Represented by Zero Free Length Springs

Each joint type has a uniquely defined characteristic point ${ }^{12}$ in each connected body that provides a reference point for calculating the joint reaction forces in terms of the joint component relative displacements. Thus each joint is represented by two characteristic points (one in each connected body) and the manner in which these two points are separated for a specific joint type determines the joint reaction forces. For revolute (turning) joints the characteristic points are the joint centers in each connected body. For prismatic (sliding) joints 
the characteristic point in each body is the point on the sliding axis where an orthogonal line from the body's mass center intersects the axis. For a planar prismatic joint there is a reaction moment proportional to the relative rotation of the two joint component sliding axes and a normal force proportional to the separation of the two axes as measured at one of the characteristic points.

Once the characteristic points have been identified in each body, the reaction forces and moments at each joint can be written in terms of the displacements (and velocities when joint internal damping is considered) of the characteristic points. The motion of a characteristic point is purely a function of the motion of the body within which the characteristic point is fixed. Each free body in space can be described by six parameters and is generally subject to six dynamic equilibrium equations (derived from Newton's Second Law or an equivalent approach). The constraints that joints apply to the mechanical system are embodied in the reaction forces which are in turn expressed (using the ICA principles) in terms of each body's six motion parameters. Basically, as each body is added to the system to be analyzed, six unknowns and six dynamic equilibrium equations are added to the system description. No additional constraint equations are required - the ICA formulation implicitly satisfying the kinematic closure constraint relationships. (For planar problems three equations and unknowns are added by each additional body in the system to be analyzed.)

The ICA converges to a solution even when an inaccurate or unrealistic set of initial conditions are applied. Thus it can facilitate mechanism design when the designer is trying different combinations of links and joints for a particular application where only a few design characteristics are known. Thus the designer does not need to figure out consistent and accurate initial displacements and velocities for all the system parameters. Where joint stiffness values are known they can be employed. Otherwise the joints can be considered as effectively rigid by using a high stiffness value like $1 \mathrm{e} 9 \mathrm{~N} / \mathrm{m}$. When high stiffness values are used, the ICA provides solutions that are similar to those using ideal joints and kinematic closure equations ${ }^{12}$.

Once the basic principles of the ICA are understood, the dynamic analysis of mechanisms is quite straightforward. In a manner similar to the approach in undergraduate statics courses, a free body diagram is formed for each moving body. The joint reaction forces (which are functions of the body motion variables) are applied along with any external applied forces, gravity forces and d'Alembert ${ }^{1}$ (inertia) forces. The equations of equilibrium are then formed based on each free body diagram. The number of equations and unknowns will equal each other without further manipulation, so these equations can be directly numerically integrated using standard methods. The equations also have a convenient matrix form since the mass matrix is diagonal.

\section{Planar Four-bar Example}

Consider the planar four-bar shown in Figure 3. The location, with respect to a body's mass center, of each joint characteristic point located in that body is given by the polar coordinates $\left(\mathrm{r}_{\mathrm{i}}, \xi_{\mathrm{i}}\right)$ measured with respect to a coordinate system centered at the body's mass center and fixed in the body. The body-fixed coordinate system can be oriented in any way that facilitates the 
subsequent analysis. The subscript $\mathrm{i}$ identifies the particular joint characteristic point and there will be twice as many characteristic points as there are standard joints. For the planar four-bar example there will be 8 characteristic points corresponding to the 4 revolute joints. In absolute coordinates, the location of characteristic point 5 is, for example,

$$
\begin{aligned}
& \mathrm{x}_{5}=\mathrm{X}_{2}+\mathrm{r}_{5} \bullet \cos \left(\Theta_{2}+\xi_{5}\right) \\
& \mathrm{y}_{5}=\mathrm{Y}_{2}+\mathrm{r}_{5} \cdot \sin \left(\Theta_{2}+\xi_{5}\right)
\end{aligned}
$$

where $\mathrm{X}_{2}$ and $\mathrm{Y}_{2}$ define the absolute location of the mass center for body 2 , and $\Theta_{2}$ is the CCW rotation of body 2's body-fixed coordinate system. In this example, if link 2 has its mass distributed symmetrically with respective to the line between its revolute joints, the body-fixed coordinate system $x$-axis can be lined up with the coupler link line shown and $\xi_{5}$ will be zero and $\xi_{4}$ will be $\pi$. With the same symmetric mass distribution assumption, the other characteristic point $\left(\mathrm{x}_{\mathrm{i}}, \mathrm{y}_{\mathrm{i}}\right)$ coordinate pairs can be similarly defined. For the planar four-bar, with each bodyfixed coordinate system $x$-axis lined up with a line connecting each body's revolute joints, the moving link $\xi_{\mathrm{i}} \mathrm{s}$ will be either $0(\mathrm{i}=3,5,7)$ or $\pi(\mathrm{i}=2,4,6)$. If the fixed link $\mathrm{d}$ is horizontal as shown and its coordinate system is chosen to be coincident with the absolute coordinate system, which located at the ground-crank revolute joint, then $\xi_{1}=\xi_{8}=0$. The $\Theta_{\mathrm{i}}$ s define the absolute angle of rotation of each moving link. If the mass center of each link is also at the link mid-point, then $\mathrm{r}_{1}=0, \mathrm{r}_{2}=\mathrm{r}_{3}=\mathrm{a} / 2, \mathrm{r}_{4}=\mathrm{r}_{5}=\mathrm{b} / 2, \mathrm{r}_{6}=\mathrm{r}_{7}=\mathrm{c} / 2$ and $\mathrm{r}_{8}=\mathrm{d}$.

Applying Newton's Second Law in 2-D form to body 2 yields

$$
\begin{aligned}
& \mathrm{k}_{3} \cdot\left(\mathrm{x}_{6}-\mathrm{x}_{5}\right)-\mathrm{k}_{2} \bullet\left(\mathrm{x}_{4}-\mathrm{x}_{3}\right)=\mathrm{m}_{2} \cdot \mathrm{X}_{2} \prime \\
& \mathrm{k}_{3} \cdot\left(\mathrm{y}_{6}-\mathrm{y}_{5}\right)-\mathrm{k}_{2} \bullet\left(\mathrm{y}_{4}-\mathrm{y}_{3}\right)=\mathrm{m}_{2} \cdot \mathrm{Y}_{2}^{\prime \prime} \\
& -\mathrm{k}_{3} \bullet\left(\mathrm{x}_{6}-\mathrm{x}_{5}\right) \bullet \mathrm{r}_{5} \bullet \sin \left(\Theta_{2}+\xi_{5}\right)+\mathrm{k}_{3} \bullet\left(\mathrm{y}_{6}-\mathrm{y}_{5}\right) \cdot \mathrm{r}_{5} \bullet \cos \left(\Theta_{2}+\xi_{5}\right) \\
& +\mathrm{k}_{2} \bullet\left(\mathrm{x}_{4}-\mathrm{x}_{3}\right) \bullet \mathrm{r}_{4} \bullet \sin \left(\Theta_{2}+\xi_{4}\right)-\mathrm{k}_{2} \bullet\left(\mathrm{y}_{4}-\mathrm{y}_{3}\right) \bullet \mathrm{r}_{4} \bullet \cos \left(\Theta_{2}+\xi_{4}\right)=\mathrm{J}_{2} \bullet \Theta_{2}{ }^{\prime \prime}
\end{aligned}
$$

where $\mathrm{k}_{\mathrm{i}}$ is the radial stiffness of joint $\mathrm{i}, \mathrm{m}_{2}$ is the body's mass, $\mathrm{J}_{2}$ is the body's polar mass moment of inertia, and the " symbol represents the second derivative with respect to time for the preceding variable. Similar equations can be written for body 1 and body 3 so there is overall a set of nine ODEs in the nine variables $X_{i}, Y_{i}, \Theta_{i}, i=1,2,3$. These equations include the effects of the joint reaction forces and inertia forces. The effects of any external applied loads are just added as additional terms to these nine equations and no further equations are required to describe the system. The equations can be numerically integrated starting from a set of initial conditions. To avoid having to use very small time steps for numerical integration, the integration algorithm selected should be one that is designed to work on ODEs that are considered to be numerically stiff ${ }^{3}$. MATLAB ${ }^{13}$ and MATHCAD ${ }^{14}$ have built-in functions that meet this requirement. 


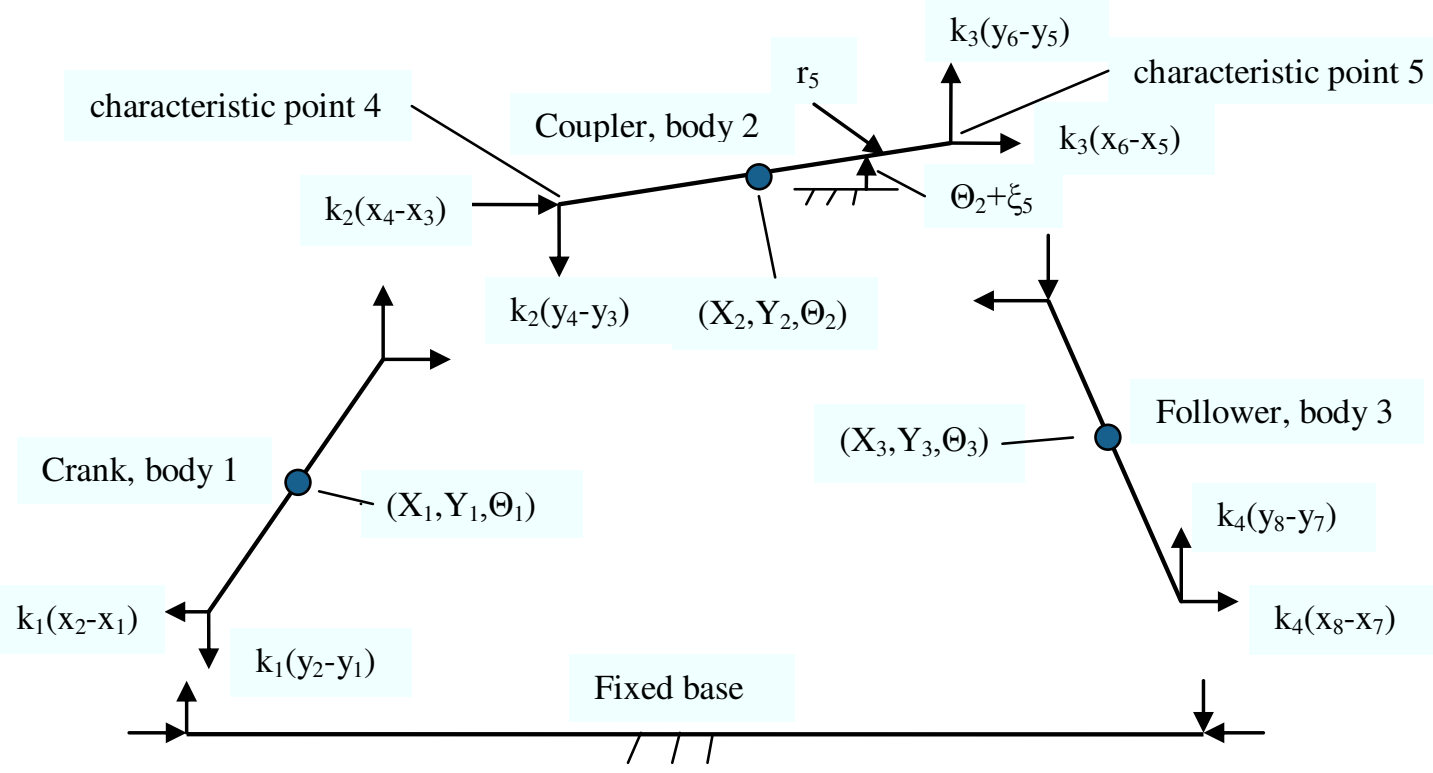

Figure 3 Planar Four-bar ICA Variables

As a sample numerical case, consider a planar four bar with a viscous rotational load of $10 \mathrm{~N}$ $\mathrm{m} / \mathrm{s}$ acting on body 3 and a $2 \mathrm{KW}$ nominal $1800 \mathrm{rpm}$ induction motor (reduced by a 10:1 ratio gear train) acting on body 1 . The four-bar has a crank length of 0.1 meter, a coupler and follower of lengths 0.4 meters each and a base length of 0.6 meters. The crank has a mass of 1 $\mathrm{kg}$ and $\mathrm{J}$ of $100 \mathrm{~kg}-\mathrm{m}^{2}$. (The high crank $\mathrm{J}$ is because a flywheel has been included with the combined crank, motor and gear train mass moments of inertia.) The coupler and follower each have a mass of $4 \mathrm{~kg}$ and a J of $0.12 \mathrm{~kg}-\mathrm{m}^{2}$. The bearings have a nominal commercial bearing radial stiffness of $5 \mathrm{e} 8 \mathrm{~N} / \mathrm{m}$. except for the coupler link bearings, which are rubber mounted so their effective stiffness is $5 \mathrm{e} 6 \mathrm{~N} / \mathrm{m}$.

For this example the nine equations of motion for the planar four-bar were numerically integrated using MATLAB [13] ODE solver ode15s for 1 second of motion. The results are the moving link mass center displacements and velocities and the link angular displacements and velocities as a function of time. The joint reaction forces can be directly calculated from the motion parameters. For example the horizontal joint reaction force at characteristic point 5 (Figure 3 ) is given by $\mathrm{F}_{65 \mathrm{x}}=\mathrm{k}_{3}\left(\mathrm{x}_{6}-\mathrm{x}_{5}\right)$ where $\mathrm{x}_{5}$ is computed from the numerical integration results using Eqn. (1) and $\mathrm{x}_{6}$ is similarly calculated.

Figure 4 shows that the variable inertia of the system causes the crank and input induction motor velocity to slightly fluctuate. The constant voltage torque speed characteristics of the drive motor are included in the model with the assumption that the torque-speed relationship is linear in the range $1700-1900 \mathrm{rpm}$, the applied torque is a maximum at $1700 \mathrm{rpm}$, the applied torque is zero at $1800 \mathrm{rpm}$, and the motor acts as a brake/generator at speeds greater than 1800 rpm. Figure 5 shows that the motor velocity fluctuations in Figure 4 are in synch with the follower velocity fluctuations. Also Figure 5 shows that the abrupt startup, the initial conditions which are only approximately consistent, and the bearing flexibility lead to an initial transient in the follower angular velocity. Other than the initial transient, the ICA results for this example are 
similar to the results from a conventional approach using exact initial conditions and ideal joint behavior.

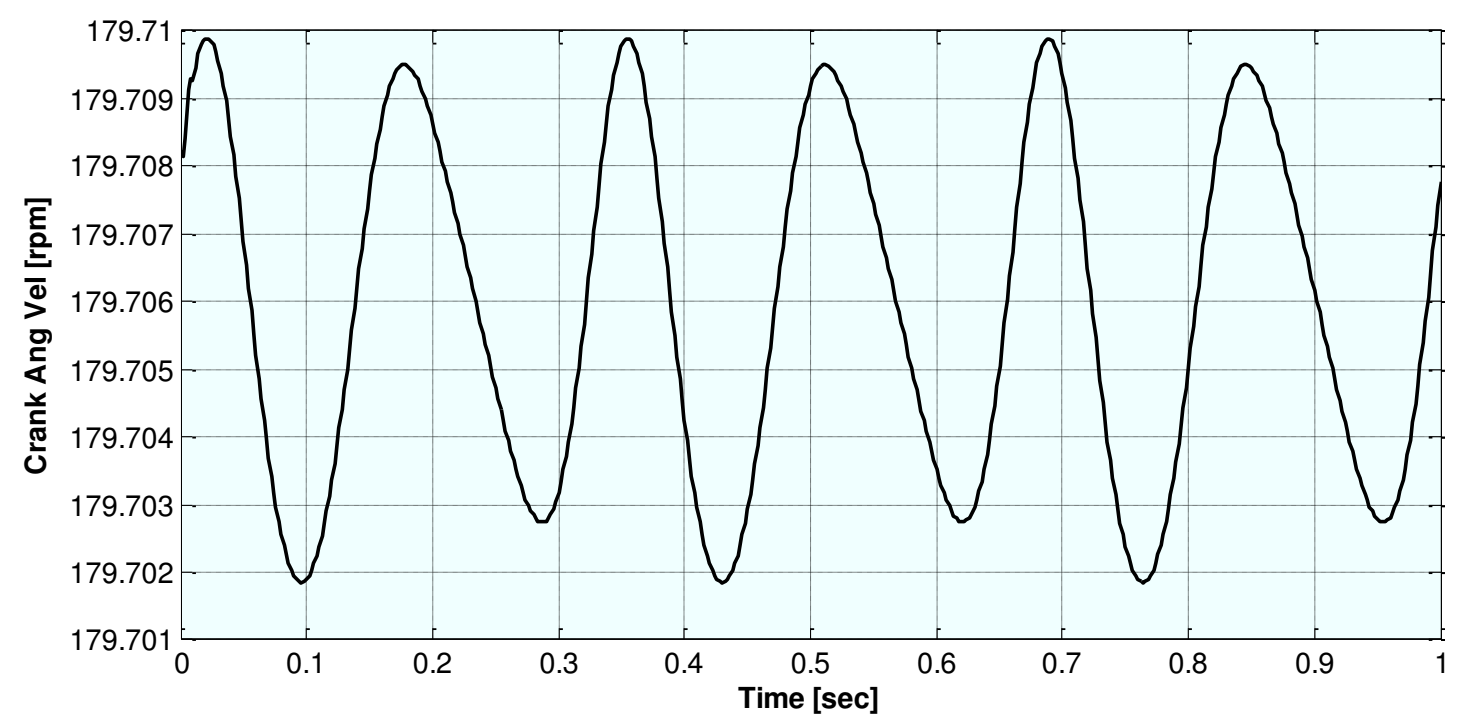

Figure 4 Planar Four-bar Crank Angular Velocity

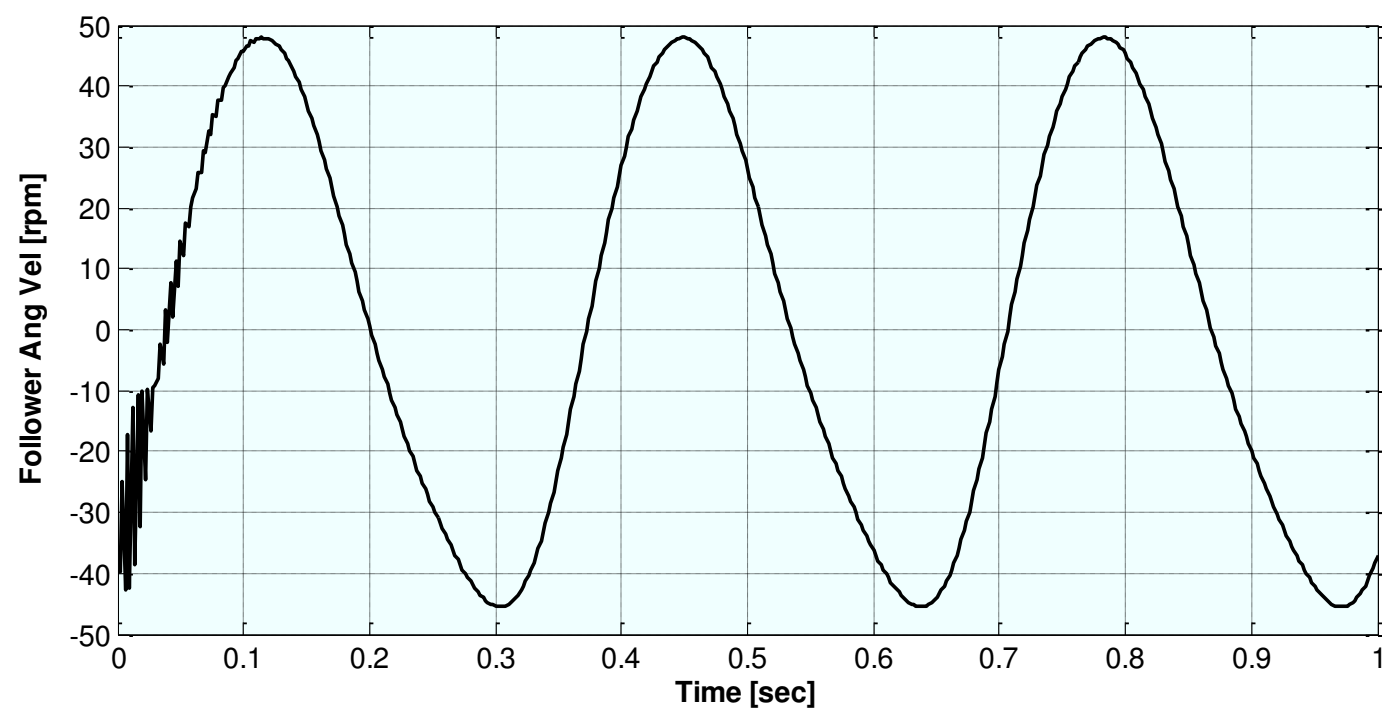

Figure 5 Planar Four-bar Follower Angular Velocity

\section{Vehicle Front Suspension Example}

Figure 6 is a photograph of the front suspension of a 2008 Toyota Tundra. Figure 7 is a schematic representation of this suspension. In addition to the fixed ground member, the suspension has 7 moving bodies including the truck body. An eighth zero mass moving body with a horizontal prismatic joint connecting it to the ground (and a revolute joint connecting it to the tire at the contact patch center) needs to be added at the ground contact patch of the right wheel to allow lateral sliding of that wheel with no resistance. This lateral free motion along the ground corresponds to the situation where the vehicle is travelling freely down the road. (When 
the vehicle is stationary there is considerable lateral resistance due to friction between the tires and the ground.) The other wheel is attached to the ground with a revolute joint at the center of its contact patch. This left-right distinction is arbitrary and the ground contact conditions could be reversed with no change in the analysis results. The other 8 joints can be considered revolute joints. The suspension is held in its neutral position by preloaded springs which are located on the same strut axes with the shock absorbers.

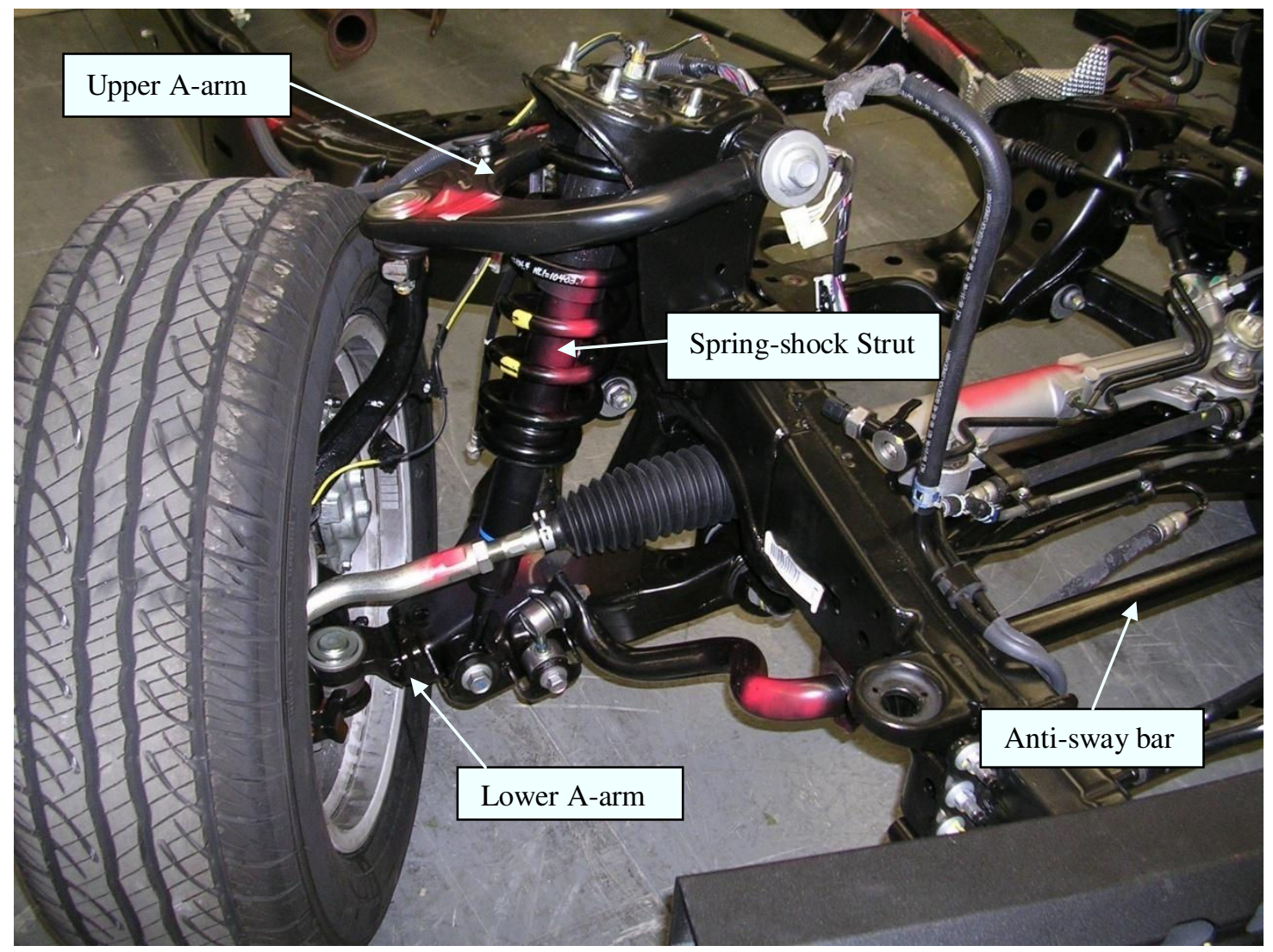

Figure 6 Tundra Front Suspension

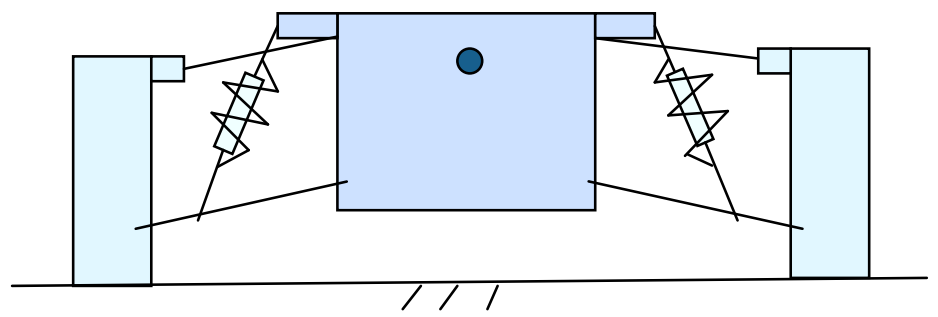

Figure 7 Schematic of the Tundra Front Suspension

Figure 8 is a screen shot of the GUI input dialog that describes this suspension using the author's program ICAP. ICAP is a program written in MATLAB that uses the ICA to solve 
planar mechanism problems. ICAP also has an automated GUI to support plotting of the dynamic simulation results (Figure 9) or the user can further manipulate the resulting data (saved by ICAP in a .MAT file) using standard MATLAB commands.

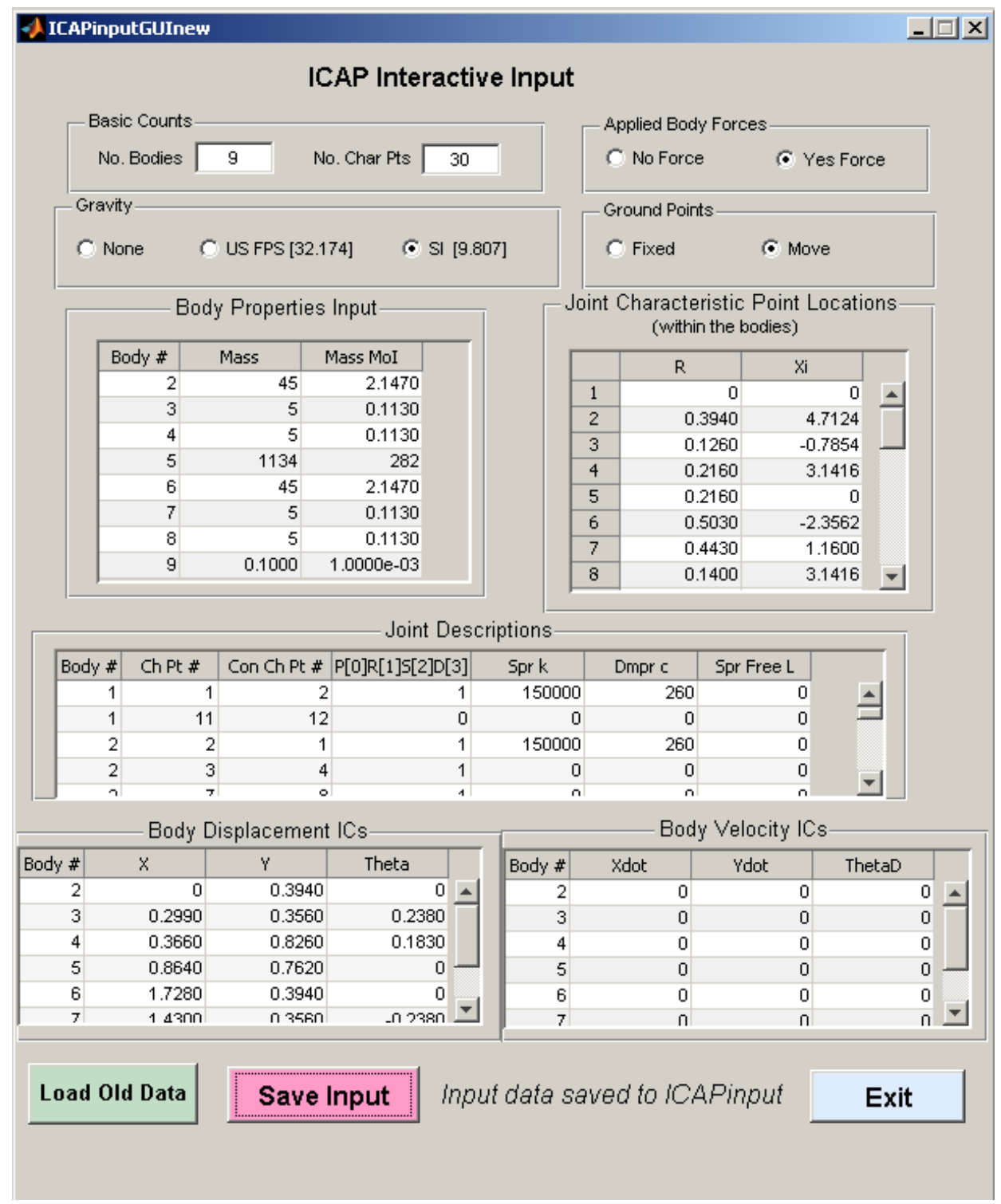

Figure 8 ICAP Input GUI for the Tundra Front Suspension

Figure 9 shows the vertical component of the force acting on the left lower arm to body joint when travelling over a $0.1 \mathrm{~m}$ jump in road surface elevation. The solid line shows the response when all the suspension joints are rigidly mounted with a nominal radial stiffness of $1 \mathrm{e} 8 \mathrm{~N} / \mathrm{m}$. The dotted line shows the response when the A-arm to body joints are all rubber mounted with a radial stiffness of $5 \mathrm{e} 5 \mathrm{~N} / \mathrm{m}$. The use of rubber mounts does not reduce the magnitude of the bearing force. However the rubber mount response does not have the high frequency oscillations superposed on its fundamental response as is the case for the stiff mounting. These force 
oscillations for the stiff mount system might excite a component in the truck body that generates a noise objectionable to the truck's human occupants.

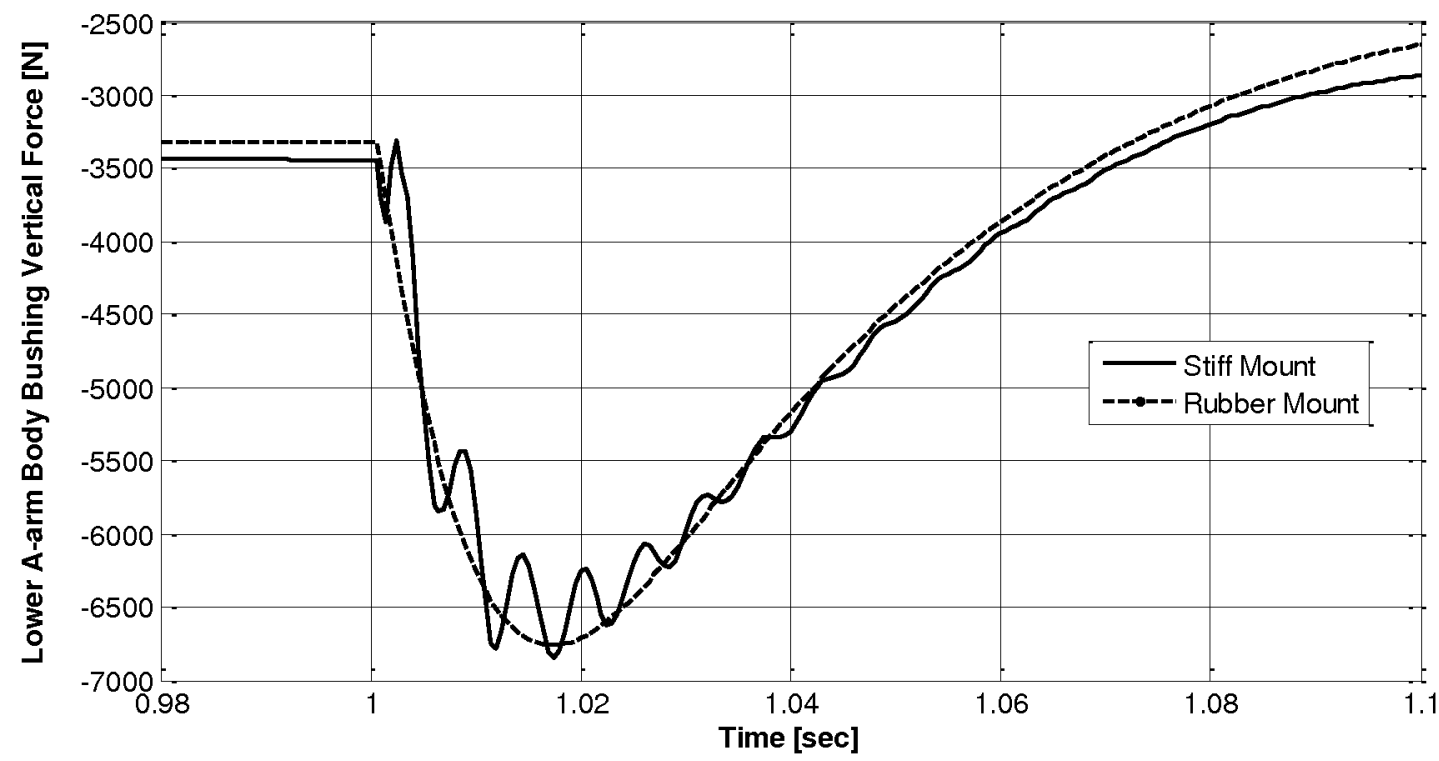

Figure 9 Bushing Vertical Force Response to an Abrupt Road Surface Change

Kinds of Problems Solvable Using the ICA

The ICA provides an alternative method for teaching students how to develop a system to control the motion of a body. Currently students learn about mechanical motion control by analyzing successively more complex devices starting with a lever, and then a planar four-bar, slider crank, planar six bar, and then some simple spatial linkages and manipulators. With each device the ever-more complex closure constraint equations, and the specialized methods to deal with them, are formed and studied. This is a difficult learning experience and particularly discourages the average student from considering analyzing or designing anything but the simplest mechanism in their future. With the ICA, students and designers can fully analyze complex mechanisms using the same free body diagram approach they learned in statics. This changes the learning emphasis from sophisticated mathematical manipulation to further engaging the design issues and underlying physics for the proposed mechanism.

The ICA can be used to analyze the dynamics of any open or closed loop mechanisms where members can be considered as rigid bodies. It is particularly valuable for problems where

- mechanism joint mounts are flexible (typically using rubber);

- a joint bearing surface is a plastic material;

- when a designer has reason to use a joint with specific compliance characteristics;

- or when a designer is rapidly evaluating different mechanism configurations for a prototype and does not want to spend time evaluating consistent initial conditions.

The basic stiffness and damping characteristics of specific joints can be easily changed in an ICA model. This enables easily observing mechanical system behavior changes such as when a specific joint's behavior changes from being a low-pass filter that solves a vibration problem to 
being a mechanical oscillator that is the source of a vibration problem. The ICA and ICAP are thus basic tools that enable students and designers to more simply examine integrated mechanism design and vibration problems.

\section{Conclusion}

The ICA provides a straightforward alternative method to determine the equations that describe the motion and forces in a mechanical system consisting of rigid bodies connected by standard joint types. One can visualize the ICA as using springs with certain characteristics (corresponding to the joint type) to connect the bodies in the system being analyzed. Irrespective of the initial conditions, these special springs will force the mechanism towards a minimum potential configuration that corresponds to a completely assembled mechanism. The dynamic equilibrium conditions can be written for each moving body using Newton's second law, where the joint reaction forces are expressed in terms of the six (or three for planar problems) basic kinematic coordinates for each rigid body. The equations and unknowns for the problem are simply six times the number of moving bodies (or three times for planar problems). Standard ODE numerical integration routines can be used to solve the equations. The ICA can be applied to problems where the joints behave ideally due to their high stiffness or to problems where the joints are designed to be more flexible. The solution approach is the same in either case and is generally simpler to implement than the conventional approach that uses kinematic loop equations to constrain the basic dynamic equilibrium equations.

The ICA lends itself to being computer automated to handle arbitrary mechanism topologies. The author has written such a program (using MATLAB) that can solve for the kinematic properties and joint reaction forces for a planar mechanism of arbitrary complexity. The mechanism can have multiple open and closed loops. GUIs are used to initiate the program, provide the mechanism description and operating conditions and identify the outputs of interest. The vehicle front suspension problem illustrates the use of this computer program.

\section{References}

1. Norton, R. L., Design of Machinery, McGraw-Hill, 2008.

2. Garcia de Jalon, J. and Bayo, E., Kinematic and Dynamic Simulation of Multibody Systems, Springer-Verlag, 1994.

3. Ascher,U. M. and Petzold, L. R., Computer Methods for Ordinary Differential Equations and DifferentialAlgebraic Equations, Society for Industrial and Applied Mathematics, 1998.

4. Chace, M. A., "Analysis of the Time-Dependence of Multi-Freedom Mechanical Systems in Relative Coordinates", Transactions of the ASME, Journal of Engineering for Industry, Vol 89, No 1, Feb 1967, pp 119125.

5. Baumgarte, J., "Stabilization of Constraints and Integrals of Motion in Dynamical Systems", Computer Methods in Applied Mechanics and Engineering, Vol 1, 1972, pp 1-16.

6. Ascher,U., Chin, H., Petzold, L., and Reich, S., "Stabilization of Constrained Mechanical Systems with DAEs and Invariant Manifolds", Journal of Mechanics of Structures and Machines, Vol 23, 1995, pp 135-158.

7. MSC Adams, MSC Software Corp., Santa Ana, CA.

8. Reuleaux, F, The Kinematics of Machinery, MacMillan, 1876 (translation by A. Kennedy of 1875 German text); also Dover, 1964. 
9. SimMechanics, MathWorks, Natick, MA.

10. Working Model 2D, Design Simulation Technologies, Canton, MI.

11. Marsh, E.R., and Yantek, D.S., "Experimental Measurement of Precision Bearing Dynamic Stiffness", Journal of Sound and Vibration, 201(1), 1997, pp 55-66.

12. Sutherland, G., "Mechanism Analysis Using Implicit Constraints", Proceedings of the ASME International Design Engineering Technical Conferences, August 2008.

13. MATLAB, MathWorks, Natick, MA.

14. Mathcad, Parametric Technology Corp., Needham, MA. 